\title{
DIGITAL TECHNOLOGY The evolving role of the radiologist
}

\author{
Author: Huw Walters ${ }^{\mathrm{A}}$
}

The rise of artificial intelligence (AI) in diagnostic radiology has raised fears for the future of the radiology workforce, but on the contrary it opens an exciting new frontier for the specialty. Taking two examples: Stanford's ChexNet software boasts the ability to detect pneumonia on chest $\mathrm{X}$-rays more reliably than consultant radiologists, the first 'superhuman' software to achieve this; while Qure's qER triages head computed tomography to ensure that those most likely to contain serious pathology are pushed to the top of the reporting pile. These types of software have the potential to improve the prioritisation of tasks to allow radiologists to work more safely and effectively. Taking a synergistic approach offers the opportunity to improve patient care by optimising human workflow and workload.

While AI could bring about huge progress in acute radiology reporting, the rise of the machines will bring its own complications. Human pathology is variable, and no matter how accurate future generations of AI become, it will still be fallible. What then, when this software makes a mistake? Human error is usually random, but computer error is systematic, and those mistakes will be repeated by the software until corrected. The 'black box' problem of AI makes correcting misinterpretation even harder; developers often lack full understanding of how the software translates data input into reports. Systematic and ingrained error would open developers up to costly litigation, meaning software companies may be unwilling to take on full legal responsibility for reporting accuracy. This either leads to clinicians dealing with useless 'cannot exclude' reports, or requires AI to be integrated into a radiologist-led system.

The hopes that AI will radically transform all of healthcare may be looked back upon as akin to the claims in the 1890s that X-rays would soon be used to project knowledge directly into medical students' brains. In looking forward to the future of radiology, fears of AI making radiologists redundant are perhaps overblown. In fact, the future radiologist may combine cutting-edge technology with the art of medicine to improve patient care, in a new form of bionic working.

Address for correspondence: Dr Huw Walters, Royal Free Hospital, Pond Street, London NW3 2QG, UK.

Email: huw.walters@nhs.net 